\title{
Introduction: space-time and the wave function
}

\author{
Albert Solé ${ }^{1}$ - Carl Hoefer ${ }^{2}$
}

Published online: 18 July 2015

(C) Springer Science+Business Media Dordrecht 2015

All the papers in this special issue deal with the questions of what the wave function represents and what the implications of quantum realism are in relation to our conception of space. These questions were the basis of the discussions that took place over two conferences which, under the title of Space-time and the wave function, were held in Barcelona in April 2013 and May 2014. The papers that are included here-or preliminary versions of them-were presented and discussed at those conferences. ${ }^{1}$

There have been several conferences dedicated to this theme, together with various papers published and this is the first special issue dedicated to the subject since the appearance of the important book edited by Ney and Albert (2013). In our opinion, three fundamental developments in the literature have contributed to opening up the debate that we are concerned with here in the current terms and they have had a notable influence on many of papers in this volume. There is no better introduction to the issue than to briefly comment on these developments.

The first and most evident of those precedents is Albert's spirited defence of realism about configuration space almost two decades ago (Albert 1996). In order to understand Albert's argument—which was what really triggered the whole debate-some

\footnotetext{
${ }^{1}$ With the exception of the paper by Dorato who was invited to participate in the second conference but was not able to attend and present his work for personal reasons.

$凶$ Albert Solé

albert.sole@ub.edu

Carl Hoefer

carl.hoefer@gmail.com

1 Departament de Lògica, Història i Filosofia de la Ciència, Universitat de Barcelona, Carrer Montalegre 6, 08001 Barcelona, Spain

2 ICREA Research Professor, Departament de Lògica, Història i Filosofia de la Ciència, Universitat de Barcelona, Carrer Montalegre 6, 08001 Barcelona, Spain
} 
preliminary caveats are necessary concerning the wave function: the real star of this special issue. $^{2}$

In the first place, we need to consider that, mathematically, the wave function of a system is a complex field defined in the so-called configuration space of the system. ${ }^{3}$ In other words, the wave function assigns a complex number to each point of that space. The notion of "configuration space" is borrowed from classical mechanics. In that theory, if we have a system of $N$ particles, it is sometimes convenient to represent the positions of all the particles through a unique point $Q \equiv\left(\boldsymbol{Q}_{1}, \boldsymbol{Q}_{2}, \ldots, \boldsymbol{Q}_{N}\right) \in$ $\mathbb{R}^{3 N}$, where $\boldsymbol{Q}_{i} \in \mathbb{R}^{3}$ are the position coordinates in physical three-dimensional space of the $i$ th particle. Configuration space is the set of all points that-like $Q-$ represent a possible configuration of all the particles of the system in physical threedimensional space and it trivially follows that configuration space is $3 \mathrm{~N}$ dimensional. Going back to quantum mechanics, the fact that the wave function of a system is defined in configuration space amounts to the following. If we have a one-particle system, its wave function assigns a number to each point of the ordinary, three-dimensional space. In this respect, the wave function of a one-particle system can be thought of as analogous to a classical field. If we have a two-particle system, however, then six spatial coordinates-and not only three-must be specified in order to specify the value of its wave function. In general, for an $N$-particle system, the specification of $3 N$ spatial coordinates is required to specify the value of its wave function.

Classically, configuration space is considered to be a space of states (or a subspace of the space of states) since each point in it represents one possible state of the system under consideration. Therefore, configuration space is interpreted in classical mechanics as a representational construct whose introduction is due to its practical usefulness, but not as a physical space. As we will see below, Albert and others who are realists with regard to configuration space maintain that, if we take quantum mechanics seriously, we must assume that configuration space is the fundamental physical space and not a space of states. ${ }^{4}$

Another important preliminary consideration has to do with entanglement. Imagine two quantum systems that are entangled. It turns out that there is no way to assign a

\footnotetext{
${ }^{2}$ For more details than we can offer here, see the introduction of Ney and Albert (2013).

3 In quantum mechanics the state of a physical system is represented by a vector (strictly speaking, a ray) in the corresponding Hilbert space which, for systems with continuous degrees of freedom, has an infinite number of dimensions. When a specific basis of Hilbert space is chosen, the projections of the vector on each one of the axes of the basis allow us to define a complex function. This function is, in fact, the wave function. If the basis chosen is that defined by the position eigenvectors, the wave function is mathematically a complex field in configuration space. In his seminal paper, Albert is implicitly assuming this particular choice of basis, which may be considered the most natural in the context of non-relativistic quantum mechanics.

4 As Maudlin (2010) rightly notes, the very notion of "configuration space", as borrowed from classical mechanics, presupposes the existence of a lower dimensional space in which some particles are configured. In this respect, if one assumes with Albert that configuration space is the fundamental physical space, and that there is no space of lower dimension with particles in it, then referring to this fundamental space as configuration space is clearly misleading. It would also be wrong to consider that the dimensionality of the fundamental space depends on the number of particles in the universe if, fundamentally, there are no particles but just a field. In this respect, we consider that the configuration space realist must take the dimensionality of the fundamental space as a brute fact about our world.
} 
wave function to each individual system such that, through knowledge of those wave functions, we could infer all the properties of the whole combined system. ${ }^{5}$ In general, the wave function of the combined system is the only one that obeys the dynamical laws of the theory and the only one that allows us to determine and make predictions regarding all the properties of the systems involved, including relational properties. ${ }^{6}$ So, in quantum mechanics, we have a type of holism: the wave function of the whole takes priority over the wave functions of the parts, which are not always well defined and — when they are — depend on the former. This leads us to the necessary distinction between the universal wave function (the wave function of the entire universe) and the wave functions of arbitrary sub-systems of the universe, and to conclude that, in terms of establishing a quantum ontology, the primary candidate for reification must be the universal wave function and not the wave functions of sub-systems of the universe.

In his article, Albert aims to elucidate the ontological consequences of nonrelativistic quantum mechanics. He starts from the assumption that our universe is governed by the laws of that theory and assumes that its Hamiltonian has the following form:

$$
\begin{aligned}
\mathrm{H}= & \sum_{i=1}^{\mathrm{N}}\left(\boldsymbol{p}_{i}\right)^{2}+\sum_{\substack{j, k=1 \\
j \neq k}}^{\mathrm{N}} \mathrm{V}_{i j}\left(\left[\left(q_{3 j-2}-q_{3 k-2}\right)^{2}+\left(q_{3 j-1}-q_{3 k-1}\right)^{2}\right.\right. \\
& \left.\left.+\left(q_{3 j}-q_{3 k}\right)^{2}\right]^{1 / 2}\right)
\end{aligned}
$$

For a Hamiltonian such as (1), the solutions of the Schrödinger equation,

$$
i \hbar \frac{\partial \Psi(q, t)}{\partial t}=\mathrm{H} \Psi(q, t)
$$

are wave functions $\Psi(q, t)$ defined in the configuration space of the universe, which has $3 N$ dimensions.

Albert is well aware that defenders of one or other quantum theory would disagree about whether the wave function provides a complete representation of a physical system, and about whether the evolution given by the Schrödinger equation (2) is universally valid. However, everyone would agree that the wave function is the fundamental theoretical term of quantum mechanics and that, through Born's rule, the wave function allows us to derive the empirical predictions of the theory. ${ }^{7}$ Therefore, Albert does not doubt that a committed quantum realist must reify the wave function. If, in mathematical terms, the universal wave function is a field defined in a $3 \mathrm{~N}$-dimensional configuration space, then the most natural realist interpretation (and the only interpre-

\footnotetext{
5 That is, the states of the two systems are non-separable.

${ }^{6}$ For a discussion of entanglement and its repercussion for wave function realism, see Ney (2015, Sect. 2).

7 The Born's rule states that the probability density for a measurement of the configuration $q$ of the particles of the system at time $t$ is given by $\rho(q, t)=|\Psi(q, t)|^{2}$.
} 
tation considered by Albert) is to assume that the wave function represents a physical field that also exists in a space of $3 N$ dimensions. Crucial to Albert's arriving to this conclusion is the fact that, given a Hamiltonian like (1), all the dynamic possibilities allowed by quantum mechanics cannot be represented by a finite set of functions defined in any space of dimension less than $3 N .{ }^{8}$ This has notorious implications since, if one requires that the fundamental physical space must be such that the physical state supervenes on intrinsic (separable) matters of fact at each point of this space, as Albert does, then the claim that configuration space is the fundamental arena of our universe seems motivated. ${ }^{9}$

Albert was not the first to adopt a realist interpretation of the wave function as a physical field in configuration space and hence to consider that configuration space itself is physically real. ${ }^{10}$ However, his paper stands out in its consideration that configuration space is the only physical space and the assumption that this conclusion is unavoidable for the quantum realist, whatever form of resolving the measurement problem she chooses. Although Albert's position is commonly dubbed as "wave function realism" in the literature, we find this terminology rather inappropriate because, as we will see, there may be other prima facie realist interpretations of the wave function not amounting to the claim that it represents a physical field in configuration space. In this regard, we prefer to dub "configuration space realism" the metaphysical thesis that configuration space exists as a physical space. Moreover, we refer to the thesis that the wave function represents a physical field in configuration space as "wave function field realism". If one thinks, in addition, that the wave function stands for the only physical object, we then have "wave function monism", which turns into "wave function field monism" if this object is a field in configuration space.

Assuming wave function field monism raises two questions that, although related, are different but are not always distinguished. In the first place, we can ask in what way the objects of our everyday experience — cats, tables, etc.—can be found in the wave function. To put it another way, we need to establish an account of reduction between the objects of our experience and the fundamental ontology. ${ }^{11}$ In the second place, given that the fundamental space is $3 N$-dimensional, we can ask in what way the three-dimensional character of the objects of the experience is recovered-if this is indeed recovered. In his paper of 1996, Albert defends that the macroscopic objects

\footnotetext{
8 In Albert's own words: "for any co-ordinatization whatever of configuration space, the set of all trajectories of a quantum-mechanical world with Hamiltonian (1) will include trajectories which pass though states that are completely non-separable in those co-ordinates, states which cannot be expressed as products of functions of any proper subset of those co-ordinates (adapted from Albert 1996, fn. 8). For a reflection on the connection between non-separability and configuration space realism and an assessment of Albert's argument, see Myrvold (2015, Sect. 3).

9 For a reflection on the connection between non-separability and configuration space realism and an assessment of Albert's argument, see Myrvold (2015, Sect. 3).

10 Bell's (1987) endorsement of wave function field realism in the context of Bohmian mechanics is well known and much cited. Other Bohmians, such as Valentini (1992), Holland (1993) and Bohm and Hiley (1993) have defended a similar position.

11 The relation of reduction established would depend, naturally, on whether configuration space is inhabited only by the wave function, or whether it is considered that there are more physical objects in it.
} 
that we experience have a merely apparent character and he tells a story of how those appearances originate, based on the form of the Hamiltonian of the universe (1). ${ }^{12}$

Albert's paper generated numerous reactions, the majority of them critical of his argument in favour of the reality of configuration space. ${ }^{13}$ Although the papers that are presented here are not directly critical of Albert's argument, many of them do represent indirect criticism. To the extent that one defends a non-instrumentalist (and hence realist) interpretation of the wave function without insisting on the reality of configuration space, one is in fact criticising Albert's position. There are many proposals of that type from the Bohmian camp-but not only from there-and several are explored in this special issue.

However, before considering Bohmian mechanics, we want to mention the work of philosophers such as Deutsch, Wallace and Saunders, in order to clarify and try to resolve some of the problems that arise in the context of Everett's interpretation (1957), converting it into a many-worlds theory. ${ }^{14}$ Taken together, that work constitutes precisely the second step forward in the recent literature that has had a considerable influence on the debate that we are here considering. The Everettian considers that the wave function alone is enough to account for everything that is real and that it evolves, without exception, in accordance with the Schrödinger equation. Since that evolution is strictly deterministic, the problem arises of how to interpret the quantum mechanical probabilities furnished by Born's rule. The question becomes even more complicated when we consider that the wave function, without collapse, generally contains "branches" for each of the possible sequences of results and that all those branches are equally real, according to the Everettians. If this is so, what sense does it make to assign probabilities of measurement outcomes when all the possible results do in fact occur, according to the theory? And what can count as evidence in favour of the theory, when the theory asserts that all possible sequences of outcomes actually occur, including that which would standardly be described as disconfirming the theory? Such a theory appears to fail to meet any minimal criterion of falsifiability.

Contemporary Everettians have also attempted to resolve various ontological puzzles. To the extent that many of them consider that the wave function constitutes all that there fundamentally is, they face the same question as Albert: they have to account for how the macroscopic objects of our everyday experience are reduced to the fundamental ontology, in this case, the wave function. Wallace $(2003,2010)$ in particular tackles this question and proposes an account of reduction according to which a macroscopic object (e.g., a table) is a pattern in the structure of the wave function that is functionally identified. ${ }^{15}$ If we accept that macroscopic objects are reduced in such a way, we can

\footnotetext{
12 See Albert (1996; 279ff). Later, Albert (2013) modifies his position and suggests a non-eliminative causal account of reduction.

13 For criticism, see Monton (2002, 2006) or Maudlin (2010).

14 Concerning the many differences between these authors-the current defenders of the many worlds interpretation - and Everett himself, see the paper by Barrett in this volume (Barrett 2015). N.b., these authors tend not to use the "many worlds" phrase to label their interpretation of quantum theory.

15 Wallace's proposal regarding reduction is sufficiently flexible (or vague) and does not require the wave function to be interpreted as a physical field in configuration space. In fact, Wallace is against that interpretation and he openly opposes realism with regard to configuration space. For a discussion of Wallace's proposal, see Barrett (Sect. 6) and Ney (Sect. 5) in this special issue.
} 
literally find a world in the wave function and if, in its unitary evolution, the wave function branches suitably, duplicating patterns, then many worlds can be found in it. ${ }^{16}$ From all of that we arrive, eventually, at the fact that we do not have to postulate the existence of many worlds (as some Everettians previously did) but rather they are conceived as emerging entities, once the existence of the wave function structure is assumed.

This perspective has led some Everettians to level an argument against Bohmiansthe so-called redundancy argument — concluding that the latter are implicitly committed to a many worlds ontology. Given that the third and final recent development that we want to comment on has to do, precisely, with Bohmian mechanics, which plays a prominent role in this volume, we will now briefly describe that theory.

As is well known, in Bohmian mechanics, in order to provide a complete characterization of a physical system we need to specify not only the wave function but also the positions of the particles of the system which, according to the theory, are well defined at all times. The trajectory of the particles depends on the wave function and is given by the so-called "guidance equation":

$$
\frac{d \boldsymbol{Q}_{k}}{d t}=\left.\frac{\hbar}{\mathrm{m}_{k}} \operatorname{Im}\left(\frac{\vec{\nabla}_{k} \Psi(q, t)}{\Psi(q, t)}\right)\right|_{q=Q(t)}
$$

When it comes to elucidating the ontology of the theory, almost all those who offer an interpretation consider that $\boldsymbol{Q}_{k}$ represents the position of the $k$ th particle in a threedimensional space that corresponds with the physical space of our experience, which is taken to be fundamental. A macroscopic object such as a table is identified with a collection of Bohmian particles that, in addition to being distributed in the form of a table, must satisfy certain dynamic properties that depend on the wave function. So we see that, under this interpretation, the problem of reducing macroscopic objects to the fundamental ontology is hugely simplified and the problem of recovering the three-dimensional character of those objects becomes trivial: macroscopic objects are three-dimensional because they are collections of particles that are also threedimensional.

The interpretation of the wave function in Bohmian mechanics is, however, more controversial. The fact that the trajectory of the particles depends on the wave function in accordance with (3), has prompted some Bohmians to think that the wave function represents a physical field that 'moves' the Bohmian particles, in an analogous way to how an electromagnetic field 'moves' a charged particle that is located within it. However, given that the wave function is defined in configuration space, this proposal leads to the consideration that the Bohmian ontology consists of a field, which inhabits configuration space, and a set of particles, which inhabit three-dimensional physical space. So, we have the field and the particles in two different spaces and so the problem

\footnotetext{
16 Decoherence occurs when a quantum system interacts with its surroundings, becoming entangled with them. The idea here is that if we have a system $\mathrm{A}$ in a quantum superposition, it is almost unavoidable that an interaction is produced between A and its surroundings, so that A ends up entangled with elements of their surroundings. When such entanglement occurs, we typically are not be able to observe the distinctively quantum effects of A's superposition.
} 
arises of how the two types of entities communicate. This problem can be resolved if, as Albert (1996) proposes, we interpret $Q \equiv\left(\boldsymbol{Q}_{1}, \boldsymbol{Q}_{2}, \ldots, \boldsymbol{Q}_{N}\right) \in \mathbb{R}^{3 N}$ as the position of a single universal particle ("the marvelous point") that together with the wave function inhabits configuration space. Then, both wave and particle are in the same space and there is no longer any mystery in their communication. However, under this interpretation the problem of the recovery of the three-dimensional character of the objects of our everyday experience reappears.

Whatever the interpretation of the Bohmian particles may be, if the wave function is interpreted as a field or, more generally, as being a physical object, the proposal can fall prey to the redundancy argument mentioned above. Consider, for example, the classic case of Schrödinger's cat. ${ }^{17}$ When the wave function (which we will suppose does not collapse, as both Everettians and Bohmians assume) contains two branches, one corresponding to the live cat and the other to the dead cat, the defender of a many worlds interpretation maintains that in the structure of that wave function there are two patterns, which give rise to two emergent cats. According to the traditional story, the Bohmian judges the situation very differently. Depending on their initial positions, the Bohmian particles will evolve deterministically in accordance with (3) towards either the configuration of a live cat or the configuration of a dead cat. Therefore, it is the configuration of the particles that determines if there is a cat alive or a dead cat and, in at any given moment, there is just one cat.

The supporter of the redundancy argument will not consider, however, the situation in the same way, but will see Bohmians as accepting that the wave function is included in the ontology, just as Everettians do. Therefore, after the experiment, the Bohmian ontology (just like Everettian ontology) includes two wave function patterns that give rise to the emergence of a live cat and a dead cat, independently of what the particles do. So in this way, according to the redundancy argument, the Bohmian also has two cats and hence there are two worlds included in the Bohmian ontology. In the famous words of Deustch, "pilot-wave theories [Bohmian mechanics] are parallel-universes theories in a state of chronic denial" (1996, p. 225).

We consider that the supporters of the redundancy argument assume, without justification, that the reductive relation must be the same for both the Everettian and for the Bohmian, despite the fundamental ontology of the latter including more elements. This seems highly dubious and it would appear legitimate for the Bohmian to consider a different account of reduction, based on the particles, since particles are included in the Bohmian ontology. ${ }^{18}$ In addition, precisely the fact that particles are included in the Bohmian ontology provides resources for interpreting the Bohmian wave function in ways that are not prima facie available to the Everettian. Instead of considering that the wave function represents a physical object, it can be interpreted in relation to the particles, either as representing some property of the particles themselves or of their law-like temporal evolution. In such a case, the redundancy argument does not get off the ground, since, if the wave function itself is not interpreted as a physical object, it can hardly be claimed that its structure can give rise to a multiplicity of worlds.

\footnotetext{
17 See Schrödinger (1935).

18 For further elaboration of this point and others against the redundancy argument, see Hawthorne (2010) and Valentini (2010).
} 
Moreover, if the wave function itself is not interpreted as a physical object, the need to reify configuration space also disappears, despite it being defined mathematically in that space.

This consideration brings us to the last development in the literature that we wish to comment on in this Introduction and that has had a marked influence on the works contained in this special issue. It is the proposal of some friends of Bohmian mechanics that, in this theory, the wave function has a nomological status. ${ }^{19}$ According to this proposal, Bohmian particles, defined in three-dimensional space, form what has been called the 'primitive ontology' of Bohmian mechanics. The wave function is interpreted in analogy with a Hamiltonian, that is, as part of our representation of the law that governs the evolution of the particles.

Although there are certain analogies between the Hamiltonian and the wave function, ${ }^{20}$ the proposal that the wave function should be interpreted as a law prima facie seems counterintuitive. The wave function is a contingent solution of the Schrödinger equation (2), which is considered by many to be a genuine law of the theory. Furthermore, as a solution of that equation, the wave function has a non-trivial temporal evolution that would seem difficult to square with its supposed nomological character. In this respect, the proponents of the nomological interpretation point out that it is the universal wave function and not the effective wave function of arbitrary subsystems of the universe that should be interpreted nomologically. ${ }^{21}$ We know little as to what the form of that universal wave function might be and the supporters of the nomological interpretation demonstrate that the supposition of a static universal wave function is compatible with effective wave functions of sub-systems that manifest the desired phenomenology; that is, that have a non-trivial temporal evolution given by the corresponding Schrödinger equation and that allow to calculate the trajectories of the particles of the sub-system through the guidance relation.

The proposal of the nomological interpretation of the wave function opens up a whole range of philosophical questions, since the debate about the nature of the wave function gets entangled with the metaphysical debate about the nature of laws. This

19 This suggestion was first formulated in Durr et al. (1992) and further developed both in Durr et al. (1997) and in Goldstein and Zanghi (2013).

20 See Durr et al. (1997).

21 In Bohmian mechanics, there is a precise way to define the wave function of a given sub-system of the universe. Let A be a subsystem of the universe including S particles with position variables $x$. Let $y$ be the position variables of all the particles not belonging to A. A's conditional wave function at time $t, \psi_{t}^{\mathrm{A}}$, is defined as follows:

$$
\psi_{t}^{\mathrm{A}}(x)=\Psi_{t}(x, Y(t))
$$

where $\Psi_{t}$ is the universal wave function at $t$ and $Y(t)$ the actual configuration at $t$ of the particles in A's environment. Now suppose that the universal wave function can be decomposed in the form:

$$
\Psi_{t}(x, y)=\phi_{t}(x) \theta_{t}(y)+\Psi_{t}^{\perp}(x, y)
$$

where (a) $\theta_{t}(y)$ and $\Psi_{t}^{\perp}(x, y)$ are functions with macroscopically disjoint supports; and (b) $Y(t)$ lies within the support of $\theta_{t}(y)$. If conditions (a) and (b) are met, then $\phi_{t}(x)$ is A's effective wave function at $t$. Notice that the effective wave function of a system does not always exist but, when it does, it is equal to its conditional wave function. For an exhaustive analysis of the notion of conditional wave function, see Norsen et al. (2015), in this special issue. 
latter debate was ignored by the original proponents of the nomological interpretation; however, the connections between the two debates have been addressed recently in the literature and some papers in this special issue contribute greatly to this exploration.

There are many different views concerning the nature of laws. First of all, using Callender's ingenious expression in this volume, laws can be interpreted as bits or its. To interpret laws as "bits" is to consider that laws are not something over and above the physical objects and their natural properties, but particularly good summaries of the distributions of those properties. This is obviously what Humeans with regard to laws assume. In contrast, to interpret laws as "its" is to consider that laws do not supervene on the distribution of natural properties but are further ontological posits. This view, in turn, can be expanded in different ways. According to primitivists, laws are primitive and the actual behaviour of physical objects is accounted for, in part, because of there being some laws; which just is a brute fact about the world. According to dispositionalists, apart from natural non-modal properties, physical objects are endowed with further dispositional properties or powers and it is the existence of these properties that makes it the case that certain laws obtain. In other words, dispositionalism makes the laws real but derived, the dispositions being the truth-makers of the laws.

A question arises as to whether the nomological interpretation of the wave function in Bohmian mechanics is compatible with all these views about the nature of laws and what the benefits of each view are when applied to this particular case. If we take Bohmian mechanics into consideration - the theory that is at the origin of the nomological interpretation-Humeanism amounts to considering that the mosaic of local matters of fact contains the trajectories of the particles and that a law is whatever axiom of the system best allows us to systematize those trajectories. If Bohmian mechanics achieves the best balance between simplicity and strength, postulating a wave function that obeys the Schrödinger equation and intervenes in the guidance equation, then the wave function can perfectly well be deemed nomological in natureif it supervenes on the history of particles trajectories. A primitivist friend of the nomological interpretation would consider, in contrast, that the wave function refers to a further entity - the law — and that the Bohmian trajectories are what they are, in part, because of this law. Finally, the dispositionalist would attribute to the Bohmian particles a set of dispositions to move in such and such a way, depending on their configuration and would take the wave function to represent these dispositions.

We can see how, in Bohmian mechanics at least, the wave function can be interpreted in ways that cover all the usual categories: as a physical object in itself; as a property of a physical object; or as a law. ${ }^{22}$ Given that all these interpretive possibilities are analysed in this special issue, let us see next what the contents of the papers are.

\section{The papers}

The first paper in the volume is Jeffrey Barrett's epilogue on the Everett interpretation of quantum mechanics. In it, Barrett offers a systematic summary of the theory, mak-

\footnotetext{
22 The possibility that the wave function represents a totally new category of object (see Maudlin 2013) is not explored in this volume.
} 
ing Everett's assumptions explicit and showing how they could possibly fit together. The central thread running through the whole of the paper is the question of the theory's empirical adequacy. In Everett's theory, ideal observers are associated with determinate relative measurement records. However, according to the theory, almost every measurement yields not one, but every physically possible result; and from this, Everett concludes that no non-trivial probabilities for measurement outcomes can be defined within the theory. Given this situation, Barrett wonders to what extent Everett's theory can be empirically vindicated and discusses Everett's own views in this respect.

However, it would be an error to consider that this paper of Barrett's is just another presentation of Everett's theory. Barrett not only makes Everett's assumptions explicit, but compares them with those that supporters of the many worlds interpretation make today, in an attempt to disentangle Everett from contemporary Everettians. So, for example, Barrett discusses Everett's account of the constitution of macroscopic objects and reveals the role that decoherence plays in that account; which is very different from the role it has in other contemporary accounts. Barrett also provides a novel criticism of Wallace's position with respect to the ontological status of the wave function and analyses the proposal by contemporary Everettians to introduce probabilities into the theory, making clear here too the huge difference with Everett's original ideas.

Configuration space realists face a problem also related to the interplay between quantum mechanics and the evidence we have for it, but a problem of a very different nature from that considered by Barrett. The evidence that we use to confirm quantum mechanics consists of macroscopic objects with a certain arrangement in three-dimensional space, such as instrument needles pointing in a certain direction, spots on photographic plates, ink marks on paper, etc. Using Bell's expression, all these are local beables. If the supporters of configuration space realism consider that only configuration space and the $3 \mathrm{~N}$-dimensional objects that inhabit it are real, they will be implying that the evidence we use to confirm quantum mechanics, which consists in local beables, does not exist. We would then arrive at the conclusion that quantum mechanics is empirically incoherent, since if the theory were true, the evidence we have to believe in it would not exist.

Alyssa Ney discusses this problem in her paper; and as in many of her previous works, she defends configuration space realism through a very audacious argument. If we do not want a fundamental theory to be empirically incoherent, then whatever constitutes the evidence for the theory must be included in the basic ontology of the theory. This is what Ney calls the "overlap thesis". The typical answer, from those who support realism concerning configuration space, is to assume that this thesis is satisfied in the case of quantum mechanics since, although only the $3 N$-dimensional ontology is fundamental, there is nothing to prevent the local beables from forming part of the ontology of the theory as emerging entities which are not any less real due to their condition of being emergent. Ney sees this answer as problematic as she considers that, to date, the many and varied attempts made by configuration space realists to show that macroscopic objects reduce to the fundamental configuration space based ontology, have been unsuccessful. Does this mean that the realist with respect to configuration space is condemned to be empirically incoherent? In her paper, Ney claims that this is not the case, given that, really, the evidence that we have in favour of quantum mechanics is not three-dimensional. The stage is set for a lively argument. 
The next group of papers focus on Bohmian mechanics, which was the most widely discussed theory at both of the two conferences that were the motivation for and origin of this special issue. In the first of these papers, Travis Norsen, Damiano Marian and Xavier Oriols present a somewhat heretical Bohmian ontology that is made up solely of entities that reside in the three-dimensional space of our everyday experience; that is, local beables. The proposal of Norsen, Marian and Oriols consists of assuming that both particles and waves exist; but, instead of reifying the universal wave function in configuration space, they postulate the existence of a set of fields all of which are defined in three-dimensional space.

Since it is a straightforward consequence of the guidance equation (3) that the trajectory of each Bohmian particle depends only on its conditional wave function, one can think of these conditional wave functions as each one guiding its affiliated particle. Conditional wave functions are fields defined in three-dimensional space and the proposal here is to reify them instead of the big, universal wave function. Conditional wave functions, however, are not solutions of the Schrödinger equation but obey a non-linear non-unitary dynamics that ultimately depends on an infinite set of fields, also defined in physical three-dimensional space. In short, the price to a pay for retaining all the dynamic information contained in the universal wave function, without introducing the latter into the equations and axioms of the Bohmian mechanics, is to postulate an infinite set of three-dimensional fields whose evolution is coupled.

Norsen (2010) already explored and defended this ontology populated by an infinite number of fields. However, here Norsen, Marian and Oriols suggest a different ontological picture. They propose to cut the infinite series of coupled fields, just leaving a small number of them. In this case, the Bohmian trajectories that follow from the corresponding equations will not be exactly identical to those that would result from considering the infinite set of fields; but Norsen, Damiano and Oriols defend the notion that the discrepancy between the two sets of trajectories would be so small that the resultant theories would not be empirically distinguishable, given the current experimental margins of error. According to the authors, we would then be faced with a similar case to that of the GRW theory, ${ }^{23}$ which recognizes, for example, the possibility of macroscopic superpositions with consequences that would violate our observations, but which assigns an infinitesimal probability to such situations.

Next, in a highly suggestive paper, Craig Callender wonders whether there is any reason to reify the wave function and, to answer that question, he proposes a comparison between versions of quantum mechanics and classical mechanics, when both theories are expressed using the same formalism. Here we should point out that classical mechanics can be formulated through a formalism similar to that of Bohmian mechanics, in which classical wave functions appear. However, nobody has ever suggested reifying those wave functions, which are considered to be representational artefacts. So, to establish whether the Bohmian wave function should be reified, Callender proposes comparing it with the classical wave function, analysing the similarities and differences between the two and considering if there are any differences

\footnotetext{
23 See Ghirardi et al. (1986).
} 
that provide grounds for not reifying the wave function in the classical case but doing so in the quantum case.

Despite the classical and Bohmian wave functions having many similarities (for example, both are objects defined in spaces with a great many dimensions), Callender identifies a crucial difference: while in the classical case we do not need the wave function, in the Bohmian case the wave function is absolutely necessary in order to have a well-posed initial value problem. Although perhaps this characteristic may explain why some people have attempted to reify the wave function in Bohmian mechanics, Callender argues that this should not impress the supporter of a nomological interpretation of the wave function, at least, not one who is a Humean in regard to the nature of laws. This turns out to be Callender's favourite interpretation of the wave function; he explains how to apply it in the context of Bohmian mechanics and argues in its defence.

Mauricio Suárez embarks on a critical review of the different interpretative options that have been offered with respect to the Bohmian wave function and which-as we say above — can be summarised as interpreting it as representing a physical field, a law or a property of Bohmian particles. Suárez first rules out interpreting the wave function as a field in configuration space, due to the difficulty in explaining the supposed communication between that field (inhabiting configuration space) and the particles (inhabiting three-dimensional space). He then offers an original criticism of the nomological interpretation, based on the fact that the wave function has a non-trivial temporal evolution; and finally advocates a dispositionalist interpretation of the wave function.

Both Suárez's dialectics and his conclusions may well remind us of work by Esfeld et al. (2014). However, there are important differences between Suárez's proposal and that of those other authors. In first place, Suárez attributes a disposition to each Bohmian particle to move in some or other fashion; while Esfeld et al. defend the idea that it is only possible to attribute dispositions to the set of all the particles. So we can see that the metaphysics proposed by both parties is quite distinct. In the second place, Suárez brings into play an interpretative distinction in the field of Bohmian mechanics that Esfeld et al. do not mention. That is the division between those who claim that Bohmian mechanics is essentially a first-order theory, with the guidance Eq. (3) as the fundamental law of motion of the particles, and those who consider a version of the theory in which the law of motion is second order, with the acceleration of the Bohmian particles being proportional to the sum of the classical forces and a new quantum force that is derived from the wave function. In opposition, for example, to what Solé (2013) maintains, Suárez argues that the second-order approach—or causal approach-offers some advantages with respect to the first-order approach in relation to its explanatory power, and that precisely those advantages may come into their own when a dispositional interpretation of the wave function is considered.

Mauro Dorato also embarks on a critical assessment of the different interpretative options related to the wave function, although his objective is quite different from that of Suárez. Dorato is not aiming to present and defend a specific interpretation, but rather to evaluate the realist's situation, once all the realist interpretations of the wave function have been duly scrutinised. Dorato's conclusion is that all the viable forms of interpreting the wave function realistically share the idea that it should be understood as representing something abstract. If that is indeed the case, then a nominalist who 
rejects the existence of abstract entities, must also reject realism with regard to the wave function and maintain an instrumentalist attitude, which, after Dorato's analysis, does not seem entirely without its motivation.

Although Dorato discusses (and rejects) realism with regard to configuration space, criticising Wallace's account of reduction, the interpretation that Dorato discusses in most detail is the nomological interpretation of the wave function. Dorato leaves Humeanism to one side and analyses in depth both primitivism and dispositionalism. In this sense, Dorato's paper can be seen as complementing Callender's, as Dorato analyses those variations of a nomological interpretation that Callender does not consider. If the wave function is to be understood as a law and also as an entity, then Dorato argues that the only way to do so is to see it as a mathematical entity and, hence, abstract. The case of dispositionalism could seem different, as a disposition is not seen as an abstract entity. However, Dorato considers that due to quantum holism, in Bohmian mechanics it only makes sense to attribute a disposition to the global configuration of all the particles and, according to him, that disposition would be, once again, an abstract property.

The papers by Callender, Suárez and Dorato make an excellent prologue to the paper by Matthias Egg and Michael Esfeld. We have seen that the three former papers analyse the prospects for interpreting, within Bohmian mechanics, the wave function as a field, as a law (either from a Humean point of view, or in accordance with primitivism) or as a dispositional property. Egg and Esfeld ask whether these same interpretations constitute valid options for interpreting the wave function in the context of the GRW theory.

Egg and Esfeld's motivation stems from the idea, defended in Allori et al. (2008), that Bohmian mechanics and GRW have a common structure. We have already seen that, in Bohmian mechanics, the particles can be considered to be the primitive ontology, defined in ordinary three-dimensional space, and that the wave function can be considered to be something that has to do with the temporal evolution of that primitive ontology. Similarly, in the version of GRW considered by Egg and Esfeld, which is usually referred to as $\mathrm{GRW}_{\mathrm{m}}$, there is a primitive ontology that consists of the mass density field $m(\boldsymbol{x}, t)$ :

$$
m(\boldsymbol{x}, t)=\sum_{i=1}^{N} m_{i} \int_{R^{3 N}} d \boldsymbol{q}_{1}, \ldots, d \boldsymbol{q}_{n} \delta\left(\boldsymbol{q}_{i}-\boldsymbol{x}\right)\left|\psi\left(\boldsymbol{q}_{1}, \ldots, \boldsymbol{q}_{N}, t\right)\right|^{2}
$$

and, once again, the wave function can be understood here as having to do with the evolution of this field. ${ }^{24}$ Despite this common structure, there are important differences between $\mathrm{GRW}_{\mathrm{m}}$ and Bohmian mechanics. One of the most important is that, while Bohmian trajectories do not supervene on the wave function, with a multiplicity of trajectories being compatible with the same wave function, clearly, the mass density (4) does supervene on the wave function. Due to this and other differences between the

\footnotetext{
${ }^{24}$ In GRW, the wave function does not always evolve in accordance with the Schrödinger equation; there is an additional stochastic law that determines spontaneous collapses whose probability increases with the number of particles in the system considered.
} 
theories, Egg and Esfeld consider that neither the interpretation of the wave function as a physical field, nor the nomological interpretation, either the Humean or the primitivist version, are satisfactory in the context of $\mathrm{GRW}_{\mathrm{m}}$. According to these two authors, only an interpretation of the wave function as a stochastic disposition of the mass density to change its form is viable.

This special issue concludes with a paper which, in some way, could be seen as a methodological amendment to the papers that precede it. Note that the advocates of configuration space realism, consider this position to be a consequence of taking non-relativistic quantum mechanics seriously. In the same way, the other interpretative options in relation to the wave function discussed so far are based on theories such as Bohmian mechanics and GRW which are not relativistic theories either. In the knowledge that those theories are neither fundamental nor true, the speculative exercise that we have been engaged in is to consider what the ontological furniture of the world would be if they were. In addition, it is considered that this interpretative exercise can be performed considering only the theory in question, without taking into account from what other more fundamental theory it could be derived from.

In his paper, Wayne Myrvold rejects this approach and defends the idea that, in order to assess the meaning of the wave function, we must take into account that quantum mechanics is a non-relativistic approximation, valid solely in certain low-energy regimes, of quantum field theory. His proposal consists of analysing the non-relativistic and the relativistic versions of quantum field theory and locating the elements of those theories that, within the appropriate limits, can be identified with the wave function of non-relativistic quantum mechanics. According to Myrvold, it is through exploring the properties of those elements that we can find out about the nature of the wave function. Proceeding in this way, he challenges two ideas that are crucial for the wave function field realist. First, Myrvold shows that wave functions in quantum field theory are unlike fields because, even if they assign a value to each point of the configuration space in which they are defined, those values are not local properties of the corresponding points. Second, the configuration spaces in which wave functions are defined cannot be taken as fundamental, since they are constructed from operators defined in ordinary spacetime. It remains to be seen whether the defender of configuration space realism will accept Myrvold's methodology; but in any case, his exploration constitutes a highly suggestive counterpoint to the previous papers and an excellent close to this special issue.

Acknowledgments We would like to thank the Spanish Ministry of Economy and Competitiveness (MINECO, Research Projects FFI2011-29834-C03-03 and CSD2009-00056) for funding the two workshops that are the origin of this special issue, and the research behind this Introduction. We also wish to thank the participants in the conferences and the authors who kindly submitted their works for this special issue. And finally, special thanks to the referees who helped improve substantially the papers in this volume, and to Otávio Bueno for his patience, rigor and excellent advice throughout the editing process.

\section{References}

Albert, D. (1996). Elementary quantum metaphysics. In J. T. Cushing, A. Fine, \& S. Goldstein (Eds.), Bohmian mechanics and quantum theory: An appraisal (pp. 277-284). Dordrecht: Kluwer Academic Publishing. 
Albert, D. (2013). Wave function realism. In A. Ney \& D. Albert (Eds.), The wavefunction: Essays on the metaphysics of quantum mechanics (pp. 52-57). Oxford: Oxford University Press.

Allori, V., Goldstein, S., Zanghì, N., \& Tumulka, R. (2008). On the common structure of Bohmian mechanics and the Ghirardi-Rimini-Weber theory. British Journal for the Philosophy of Science, 59, 353-389.

Barrett, J.A. (2015). Pure wave mechanics and the very idea of empirical adequacy. Forthcoming in 'Spacetime and the wave function', Special Issue of Synthese.

Bell, J. S. (1987). Speakable and unspeakable in quantum mechanics. Cambridge: Cambridge University Press.

Bohm, D., \& Hiley, B. J. (1993). The undivided universe: An ontological interpretation of quantum theory. London: Routledge \& Kegan Paul.

Deutsch, D. (1996). Comment on Lockwood. British Journal for the Philosophy of Science, 47, $222-228$.

Durr, D., Goldstein, S., \& Zanghi, N. (1992). Quantum equilibrium and the origin of the origin of absolute uncertainty. Journal of Statistical Physics, 67, 843-907.

Durr, D., Goldstein, S., \& Zanghi, N. (1997). Bohmian mechanics and the meaning of the wave function. In R. S. Cohen, M. Horne, \& J. Stachel (Eds.), Experimental metaphysics: Quantum mechanical studies for Abner Shimony (pp. 25-38). Berlin: Springer.

Esfeld, M., Lazarovici, D., Hubert, M., \& Dürr, D. (2014). The ontology of Bohmian mechanics. British Journal for the Philosophy of Science, 65, 773-796.

Everett, H, I. I. I. (1957). 'Relative state' formulation of quantum mechanics. Reviews of Modern Physics, $29,454-462$.

Ghirardi, G. C., Rimini, A., \& Weber, T. (1986). Unified dynamics for microscopic and macroscopic systems. Physical Review D, 34, 470-491.

Goldstein, S., \& Zanghi, N. (2013). Reality and the role of the wave function in quantum theory. In A. Ney \& D. Albert (Eds.), The wavefunction: Essays on the metaphysics of quantum mechanics (pp. 91-109). Oxford: Oxford University Press.

Hawthorne, J. (2010). A metaphysician looks at the Everett interpretation. In S. Saunders, J. Barrett, A. Kent, \& D. Wallace (Eds.), Many worlds? Everett, quantum theory, and reality (pp. 144-153). Oxford: Oxford University Press.

Holland, P. R. (1993). The quantum theory of motion: An account of the De Broglie-Bohm causal interpretation of quantum mechanics. Cambridge: Cambridge University Press.

Maudlin, T. (2010). Can the world be only wavefunction? In S. Saunders, J. Barrett, A. Kent, \& D. Wallace (Eds.), Many worlds? Everett, quantum theory, and reality (pp. 121-143). Oxford: Oxford University Press.

Maudlin, T. (2013). The nature of the quantum state. In A. Ney \& D. Albert (Eds.), The wave function (pp. 126-154). Oxford: Oxford University Press.

Monton, B. (2002). Wave function ontology. Synthese, 130, 265-277.

Monton, B. (2006). Quantum mechanics and 3N-Dimensional space. Philosophy of Science, 73, 778-789.

Myrvold, W. (2015). What is a wavefunction?. Forthcoming in 'Space-time and the wave function', Special Issue of Synthese.

Ney, A., \& Albert, D. (2013). The wavefunction: Essays on the metaphysics of quantum mechanics. Oxford: Oxford University Press.

Ney, A. (2015). Fundamental physical ontologies and the constraint of empirical coherence: a defense of wave function realism. Forthcoming in 'Space-time and the wave function', Special Issue of Synthese.

Norsen, T. (2010). The theory of (exclusively) local beables. Foundations of Physics, 40, 1858-1884.

Norsen, T., Marian, D., \& Oriols, X. (2015). Can the wave function in configuration space be replaced by single-particle wave functions in physical space?. Forthcoming in 'Space-time and the wave function', Special Issue of Synthese.

Schrödinger, E. (1935). Die gegenwärtige Situation in der Quantenmechanik. Naturwissenschaften, 23(49), $807-812,823-828,844-849$.

Solé, A. (2013). Bohmian mechanics without wave function ontology. Studies in History and Philosophy of Modern Physics, 44, 365-378.

Valentini, A. (1992). On the pilot-wave theory of classical, quantum and subquantum physics. Ph.D. Dissertation, ISAS - International School for Advanced Studies, Trieste.

Valentini, A. (2010). De De-Broglie-Bohm pilot-wave theory: many worlds in denial? In S. Saunders, J. Barrett, A. Kent, \& D. Wallace (Eds.), Many worlds? Everett, quantum theory, and reality (pp. 476-509). Oxford: Oxford University Press. 
Wallace, D. (2003). Everett and structure. Studies in the History and Philosophy of Modern Physics, 34, 86-105.

Wallace, D. (2010). Decoherence and ontology. In S. Saunders, J. Barrett, A. Kent, \& D. Wallace (Eds.), Many worlds? Everett, quantum theory, and reality (pp. 53-72). Oxford: Oxford University Press. 